\title{
Acute and Chronic Limb Ischaemia
}

Andrew Nickinson MBBS (Distinction) MRCS (Eng)

George Davies Clinical Research Fellow and Higher Surgical Trainee in Vascular Surgery, University of Leicester

Matthew J Bown MD FRCS

Professor of Vascular Surgery, Department of Cardiovascular Sciences and NIHR Leicester Biomedical Research Centre.

\section{$\underline{\text { Introduction }}$}

Limb ischaemia refers to the symptomatic disruption of arterial blood flow to the extremities. The condition can be separated into acute and chronic ischaemia. Peripheral arterial disease is the leading cause of both conditions. Limb ischaemia forms a major part of the workload for vascular surgeons and is associated with significant morbidity and mortality for patients. ${ }^{1,2}$ In this review the epidemiology, aetiology and management of both acute and chronic limb ischaemia will be discussed.

\section{Chronic Limb Ischaemia and Peripheral Arterial Disease}

Chronic Limb Ischaemia is most commonly caused by peripheral arterial disease (PAD). Lower limb atherosclerosis affects over 200 million individuals worldwide. ${ }^{3}$ It is characterised by the formation of flow limiting stenotic and occlusive atherosclerotic plaques. The condition is uncommon in the under 50 age group but prevalence rises to approximately $20 \%$ in the over $80 \mathrm{~s} .{ }^{4}$ In the United Kingdom critical limb ischaemia it has been previously estimated to cost over $£ 200$ million per annum. ${ }^{5}$

PAD presents as a spectrum of symptoms from intermittent claudication through to limb threatening critical limb ischaemia (CLI). The majority of patients however are asymptomatic. ${ }^{6}$ The disease is not an isolated entity but part of a systemic atherosclerotic 
disease affecting all major arteries. Patients with PAD are therefore at increased risk of developing coronary artery, cerebrovascular and renovascular diseases. ${ }^{7,8}$

\section{Aetiology}

Risk factors for peripheral arterial disease can be classified into modifiable and nonmodifiable (Table 1).

\section{$\underline{\text { Non-Modifiable Risk Factors }}$}

Age is an established risk factor for PAD in both men and women. The condition is most prevalent amongst black ethnicities. ${ }^{9}$ Individuals with a first-degree relative suffering from symptomatic PAD have twice the odds of developing the condition compared to whose without. ${ }^{10}$ Gender and the prevalence of PAD varies depending on the economic background of the population. In high-income countries the prevalence is broadly equal between men and women, however in lower-incomes countries PAD is more prevalent in women until the age of 90 years. ${ }^{3}$

\section{Modifiable risk factors}

Hypertension and hypercholesteraemia are both well-documented risk factors and can be readily treated. ${ }^{9}$ In patients with familial hypercholesterolaemia, the prevalence of PAD is almost 10 -fold higher than the general population. ${ }^{11}$

Tobacco smoking is a leading cause of atherosclerosis. ${ }^{12}$ The prevalence of smoking within the UK has halved within the last 50 years and whilst it will be less important in the future it remains the commonest cause of PAD. ${ }^{13}$ Recently there has been a shift from traditional cigarettes towards e-cigarettes and other 'vaping' devices. ${ }^{14}$ These devices are associated with lower exposure to carcinogens and volatile organic compounds metabolites compared with traditional cigarettes, however their long-term health effects are unknown. ${ }^{15}$ 
As smoking has decreased the prevalence of Diabetes mellitus has increased and the management of diabetes is likely to play an increasing important role in the prevention and management of PAD in the future. The prevalence of diabetes within UK has almost doubled in the last 20 years and approximately $20 \%$ of patients with PAD are diabetic. ${ }^{16,17}$ The combination of macro and microvascular disease seen in diabetes is particularly damaging and amputation rates are at least over $50 \%$ higher than in non-diabetics. ${ }^{18-21}$

The association between obesity and PAD is more complicated than simply increasing body mass index (BMI). In a sample of 708 men Planas et al observed that abdominal fat distribution (waist-to-hip ratio) was associated with PAD, however BMI alone was not. ${ }^{22}$ Increasing abdominal fat distribution is a key part of the 'metabolic syndrome' which is higher prevalent in patients with PAD. ${ }^{23}$

\section{Clinical Features}

\section{Symptoms}

Intermittent claudication refers to the cramping, muscular pain which is brought on by exertion and relieved by a short period of rest. Symptoms arise at a relatively constant distance ('claudication distance') and are exacerbated by incline. The location of a patient's symptoms can help identify the level of the disease (e.g. femoropopliteal disease presents with calf symptoms). Bilateral claudication indicates the presence of aortoiliac disease. Symptom progression occurs in $25-33 \%$ of patients with claudication although the rate of subsequent major amputations is low (1-3\%). ${ }^{24,25}$

As arterial disease progresses claudication distance shorten. Limb threatening 'criticallimb ischaemia' (CLI) arises most commonly when multi-level stenotic/occlusive disease is present. This limits oxygen delivery to a level that is unable to support the basal metabolic demands of the limb.

At this point ischaemic night/rest pain occurs. Patients describe a constant, 'gnawing' pain in the extremities. By definition pain must be present for $>2$ weeks to be classified as CLI. Night-time is particularly associated with symptoms due to the loss of gravity aiding perfusion and the natural reduction in blood pressure associated with sleep. Classically 
patients are awoken with severe pain and find relief through standing or hanging the effected limb out of bed. Patients describing the need to sleep in a chair is not uncommon and this can lead to dependant oedema forming.

Limb ulceration and gangrene ('tissue loss') both occur in advanced CLI. Minor injuries can develop into frank ulceration or necrosis. Superimposed bacterial infection can rapidly lead to osteomyelitis, wet gangrene and death if not treated promptly. Given the nature of the disease the outcomes of patients with $\mathrm{CLI}$ are poor, with a $30 \%$ amputation and $25 \%$ mortality rate at just one-year following diagnosis. ${ }^{26}$

\section{Clinical Examination}

Inspection of the leg may demonstrate a pale, cool limb with evidence of hair loss, scaly skin, nail discolouration and muscle wasting. Patients with diabetes may have associated foot changes such as dry skin, toe clawing, pes cavus and peripheral neuropathy. In CLI frank necrosis and ulceration (Figure 1) may be present and care should be taken to identify wet gangrene or an underlying suppuration which both require urgent debridement. Dactylitis ('sausage digit') is indicative of digital osteomyelitis.

Palpation of peripheral pulses can help identify the level of disease, although the presence of palpable pulses does not rule out arterial disease. The abdomen should also be palpated to help identify the presence of an abdominal aortic aneurysm. Buerger's test (pallor on $<30$ degree elevation and reactive hyperaemia causing rubor on dependency) should also be completed, with a positive test indicating CLI.

Hand-held continuous-wave doppler assessment of pedal pulses is a particularly useful and readily available bed-side test, although practice is required to interpret signals. Monophasic or absent pedal signals indicate significant underlying arterial disease. Anklebrachial pressure index $(\mathrm{ABPI})$ can also be easily performed. Table 2 shows the interpretation of $A B P I$ readings. An absolute ankle pressure of $>60 \mathrm{mmHg}$ is required to heal an ulcer and pressures below this indicate CLI. Some patients have incompressible, calcified arteries which give falsely elevated and unreliable readings. This is particularly common in diabetics and in these patients toe pressures can be utilised. 


\section{$\underline{\text { Differential Diagnoses }}$}

Although PAD is the commonest cause of chronic limb ischaemia, other vascular pathologies can also cause ischaemia and should be considered.

Popliteal artery entrapment syndrome, iliac exofibrosis, cystic advential disease and fibromuscular dysplasia are all rare causes of limb ischaemia but should be considered in younger patients who lack typical risk factors for PAD. Thromboangitis obliterans (Buerger's disease) is typically seen in younger patients with a heavy smoking history. A thrombosed popliteal artery can also present with chronic ischaemia and highlights the importance of always palpating the popliteal pulse (even if pedal pulses are palpable). A persistent sciatic is a rare condition where the foetal sciatic artery fails to obliterate and the superficial femoral artery is underdeveloped. A palpable popliteal but absent femoral pulse is said to be pathognomonic of this condition.

Other non-vascular conditions may also mimic the symptoms of chronic limb ischaemia. Spinal stenosis, spinal nerve root irritation and degenerative spinal disease can all present with pain on exertion. Spinal stenosis can be differentiated from limb ischaemia as it usually eases with bending forward or walking up an incline. Spinal root irritation typically presents with numbness and paraesthesia, which is uncommon in ischaemia.

\section{Classification}

There are numerous classification systems for PAD which exist. These categorise depending on symptoms (e.g. Fontaine, Table 3), objective examination findings (e.g. Rutherford) or angiography findings. ${ }^{26}$ The Trans-Atlantic Inter-Society Consensus Document II (TASC II) system classifies depending on angiography findings. It is of particular note as it helps define treatment algorithms for different patterns of disease. ${ }^{25}$ Classification systems are useful in clinical research in order to facilitate comparison of treatments but in reality they have limited value in routine clinical practice.

\section{$\underline{\text { Investigations }}$}

Investigations aim to assess the extent of disease and help plan treatment. Duplex ultrasound, magnetic resonance angiography (MRA) and computerised tomographic 
angiography (CTA, Figure 2) are all recommended by the National Institute for Health and Care Excellence (NICE). ${ }^{27}$ The modality used will depend largely on local availability.

Duplex is an excellent first-line investigation but is operator dependant and overlying bowel gas can obscure the aorto-iliac vessels. MRA and CTA can give excellent images and are usually performed prior to planning intervention. Digital subtraction angiography (DSA) remains the gold-standard investigation and permit diagnosis and treatment of disease but is seldom used prior to other imaging.

Care should be taken in patients with renal failure or diabetes undergoing any form of angiography due to the risk contrast nephropathy. ${ }^{28}$

\section{Management}

The management of peripheral arterial disease can be divided into conservative management, revascularisation and amputation. The exact management with depend on the patient's symptoms, their fitness for intervention and the anatomy of their disease. Over-arching all of these is the modification of cardiovascular risk factors. Treatment decisions are taken as part of a multi-disciplinary team approach involving surgeons, radiologists and the patient.

\section{Risk factor modification}

Risk factor modification aims to reduce the risk of major cardiovascular events in patients with PAD. This approach is termed 'best medical therapy' (BMT) and encompasses the use of anti-platelets, cholesterol lowering medications, blood pressure control and smoking cessation. BMT should be applied to all patients with PAD and is recommended by NICE. ${ }^{27}$

Anti-platelets have been shown to reduce all cause and cardiovascular related mortality in patients with PAD. ${ }^{29}$ There is no role for anticoagulation in PAD unless the patient has another indication. In those with intermittent claudication the use of anti-platelets reduces the risk of cardiovascular mortality by $46 \%$ compared with placebo. ${ }^{30}$ Aspirin or Clopidogrel are routinely used, although the CAPRIE study showed Clopidogrel to be superior. ${ }^{31}$ Ticagrelor is routinely used in coronary disease however a recent randomised controlled study shown no superiority over Clopidogrel in PAD. ${ }^{32}$ Looking ahead, the 2018 
COMPASS trial found that the addition of low dose Rivaroxaban (2.5mg BD) to Aspirin to be superior to Aspirin alone, however this has not yet been adopted into routine clinical practice..$^{33}$

Lipid lowering medications in the form of statins are also a key part of BMT. Not only do statins reduce the risk of cardiovascular events, they have also been shown to improve claudication distance and reduce the risk of developing $\mathrm{CLI}$ and subsequent amputation. ${ }^{34,35}$ Interest is also growing in PCSK9 inhibitors (e.g. Evolocumab), a group of monoclonal antibodies which can lower LDL-cholesterol. These medications have shown exciting results in PAD and may be used more routinely in clinical practice in the near future. ${ }^{36}$

Patients with hypertension should be commenced on anti-hypertensive medications with a target blood pressure of $<140 / 80 \mathrm{mmHg} .{ }^{37}$ Smoking cessation is highly beneficial and multiple cost-effective smoking cessation methods are available. ${ }^{38}$ In those who quit, risk of myocardial infarction is returned to that of a non-smoking within 5 years of cessation. ${ }^{39}$

\section{Conservative management}

Supervised exercise is the mainstay of treatment for patients with intermittent claudication and is recommended as first-line treatment by NICE. ${ }^{27}$ Exercise involves a structured programme where the patient walks to the point of moderate discomfort, past their usual claudication distance. The therapy has been shown to increase exercise performance, although benefit to quality of life is modest. ${ }^{40}$ Supervision is a key element to the success of the intervention and yields better results than home-based therapy or simple advice. ${ }^{42,42} \mathrm{~A}$ major drawback is the limited availability of services meaning many are unable to benefit. The intervention requires patient motivation and is not suitable in those with limiting co-morbidities.

Symptomatic treatments for intermittent claudication are also available, including Naftidrofuryl. Naftidrofuryl is a serotonin 5-hydroxytrytamine 2 receptor antagonist, which has been shown to improve claudication distance compared with placebo. ${ }^{43}$ Its use in the UK is limited to patients who fail to see improvement with exercise and are not suitable for revascularisation. ${ }^{27}$ 
Conservative management has limited role in CLI and is reserved for patients who are not suitable for revascularisation. Infusion of intravenous prostanoids, such as iloprost, are on occasion used as an alternative to major amputation and are thought to improve blood flow to the extremity through a vasodilatory and anti-inflammatory effect. Evidence on their therapeutic benefits is mixed. A 2018 Cochrane review found prostanoids improve pain and ulcer-healing compared with placebo, however no improvement in amputation rates was identified. ${ }^{44}$

\section{$\underline{\text { Revascularisation }}$}

Revascularisation attempts to re-establish in-line flow to the effected limb. This can be achieved through surgery or endovascular means. Revascularisation should be considered in all patients with CLI. Early revascularisation in patients with claudication is associated with a higher risk of amputation than conservative management and is therefore usually reserved for those with disabling symptoms for whom exercise has failed to improve symptoms. ${ }^{45}$

\section{$\underline{\text { Surgical Revascularisation }}$}

Surgery can involve the use of endarterectomy (removal of the atheromatous plaque, Figure 3 ) and bypass. The principle of bypass surgery is to bypass blood from a healthy 'inflow' vessel to a distal 'target' vessel using a suitable conduit. The choice of inflow and target vessels is of critical importance to the success of the bypass.

The inflow vessel should be relatively free of disease to provide satisfactory flow into the bypass graft. The common femoral artery is usually the vessel of preference. The choice of target vessel is more complex. It should be the most proximal, disease free vessel to achieve unrestricted flow to the foot. Frequently the popliteal artery is selected if there is at least one patent tibial 'run-off' vessel distal to this which can supply the foot ('Femoralpopliteal bypass'). If the popliteal artery is diseased then the bypass is anastomosed directly to a tibial vessel ('Femoral-distal bypass'). This is usually the artery with continuous flow into the foot. In cases of CLI with tissue loss consideration should be made to select the tibial vessel supplying the vascular territory ('angiosome') of the effected part of the foot. ${ }^{46}$ 
Autologous vein is the preferred conduit for bypass surgery, providing superior long-term patency compared to prosthetic material and greater resistance to infection. ${ }^{47}$ Longsaphenous vein is conduit of choice, with arm-vein second-line. There are a variety of configurations for a vein bypass including reversed, non-reversed (with valves removed by valvotome) or in-situ technique. The method chosen will largely depend on the anatomy of the inflow/target vessels and surgeon's preference.

Prosthetic bypass grafts are used when no suitable vein exist (e.g. ePTFE, Dacron). If a below-knee bypass is required the use of a vein cuff on the distal anastomosis (e.g. Miller cuff, Taylor's patch) can help improve patency. ${ }^{48}$ Some bypass grafts are now made 'precuffed'. Recently biosynthetic xenografts grafts have become available, which combine animal and synthetic materials (e.g. Omniflow $\|^{\circledR}$, Le-Matire). These grafts are still inferior to vein but a 2017 meta-analysis did show improved patency compared with ePTFE. ${ }^{49}$

\section{Endovascular Revascularisation}

In recent years endovascular techniques for revascularisation have been increasing employed. Figures from the UK National Vascular Registry show a $71 \%$ increase in procedure numbers from 2014 to $2016 .^{2}$ The technique involves percutaneous balloon angioplasty and/or stenting of atheromatous plaque (Figure 4). The advent of subintimal angioplasty (creating a plane deep to intima to cross an occlusion) has allowed for the treatment of increasing complex occlusive disease. ${ }^{50}$ The majority of angioplasty procedures can be carried out under local anaesthetic and as a day-case, mitigating the risks of general anaesthesia and costly inpatient admission. ${ }^{2}$ This has made the procedure a particularly attractive option, especially in co-morbid patients.

There has been considerable debate regarding both surgical and endovascular revascularisation in recent years. The 2005 BASIL trial remains the only randomised controlled trial to compare outcomes of both revascularisation techniques. ${ }^{47} \mathrm{Up}$ to 2 years post revascularisation both amputation-free and overall survival were comparable. In patients surviving beyond this however surgery showed a trend towards improved amputation-free survival. ${ }^{51}$ 
Later analysis of the BASIL data also showed angioplasty had higher rate of technical compared with surgery. ${ }^{52}$ Furthermore bypass performed following failed angioplasty carried a significantly higher risk of amputation compared to primary bypass surgery..$^{53}$ It should be noted that endovascular techniques have improved since the trial was performed and therefore results may not reflect practice today.

\section{Drug eluting technology}

Recently there has been significant interest in the use of angioplasty balloons and stents coated in anti-proliferative or immunosuppressive agents (e.g. Paclitaxel). These aim at reducing restenosis which can occur following the trauma of angioplasty.

Whether this technology provides better outcomes is controversial. Although evidence indicates drug eluting balloons have superior primary patency compared with conventional angioplasty this is not translated into improved clinical outcomes for patients. ${ }^{54,55}$ Drug coated stents have also not been shown to improve patency or clinical outcomes. ${ }^{56,57}$ The results of the BASIL-3 trial should hopefully address this further. ${ }^{58}$

\section{Best revascularisation strategy}

Overall the decision on treatment should be individually tailored to the patient. An 'endovascular first' strategy is advocated in patients who are unfit for surgery or have no suitable vein. Patients who are fitter and likely to achieve independence are usually best served with bypass surgery. ${ }^{59}$ In many centres a hybrid strategy is being used, combining both open and endovascular technique. This is particularly useful to help improve inflow into a bypass.

\section{Amputation}

Major amputation is generally a last resort but can be a positive treatment option for many. Urgent primary amputation is a life-saving measure in those presenting with tracking necrotising infection or extensive necrosis. It can also provide pain relief and improved quality of life in patients with unsalvageable CLI. In patients who are nonambulatory or unfit, amputation may be a better option than attempting complex revascularisation. 
Three levels of major amputation are commonly used: below, through and above knee. The decision on level will depend on the healing and rehabilitation potential of the patient. Below knee amputation (Figure 5) provides the best change of walking ( $50 \%$ mobilise with a prosthesis compared with $25 \%$ for above knee) but adequate blood flow to the calf is required. Above or through knee amputation is a better option in patients with no chance of walking as the knee joint can develop a fixed-flexion deformity. Multidisciplinary input from specialities including specialist physiotherapists and occupational therapists is vital to aid rehabilitation.

Minor amputation are often used as an adjunct to revascularisation in patients with necrosis or deep tissue infection. The long-term effects on mobility are generally limited, although orthotics may be required to aid walking. Unless there is suppurative or spreading necrotising infection, minor amputation should take place after revascularisation.

\section{Acute Limb Ischaemia}

Acute limb ischaemia (ALI) is defined as a sudden disruption in limb perfusion that threatens the viability of the limb. ${ }^{25}$ The disease is characterised by either thrombotic or embolic occlusion of a peripheral artery and symptoms are of less than 2 weeks duration. In patients with peripheral artery disease, 'acute-on-chronic' ischaemia represents an acute deterioration in their symptoms.

Within the UK the incidence of condition is 10 per 100,000 per year. ${ }^{60}$ Despite an increasing role of endovascular techniques, the condition still represents a surgical emergency in a patient with an immediately threatened limb. Prognosis is poor with a 30day amputation rate of $30 \%$ and mortality rate of $22 \% .{ }^{61}$

\section{Aetiology}

The aetiology of ALI can be divided into thrombotic, embolic and other causes (Table 4)

\section{$\underline{\text { Thrombotic }}$}


Thrombosis is now the leading cause of ALI, largely due to the increasing prevalence of peripheral arterial disease. Thrombotic occlusions are typically due to rupture of an atherosclerotic plaque or in-situ thrombosis of a flow limiting stenotic lesions. Thrombosis of pre-existing bypass grafts or endovascular stent-grafts can also cause ALI. Frequently these events occur in the context of other illnesses, such as dehydration or malignancy.

Occlusion of a popliteal aneurysm is a cause of ALI particularly feared by vascular surgeons. The disordered flow within the aneurysm can precipitate thrombsis, which can subsequently completely occluded and worse, embolise to the tibial vessels. This situation hinders revascularisation and consequently the risk of limb loss is substantial.

In patients presenting with thrombosis of arteries free of atherosclerotic disease, underlying thrombophilia or malignancy should be considered.

\section{$\underline{\text { Embolic }}$}

Embolism was historically the leading cause of ALI, principally due to rheumatic heart disease. Emboli can consist of both platelets and cholesterol. Cardiogenic emboli account for the majority of embolic ALI events and consist mainly of platelet thrombus. This typically lodges at the bifurcation of major arteries (e.g. common femoral bifurcation). Atrial fibrillation and embolisation of a mural thrombus following myocardial infarction are common causes.

Cholesterol emboli can arise from rupture of a proximal artherosclerotic plaque or embolus from a proximal aneurysm. This can occur spontaneously but also be iatrogenic following revascularisation procedures. Cholesterol emboli can trash the small vessels of the foot causing 'blue toe syndrome' (Figure 6).

\section{Other causes}


Less common cause of ALI include trauma and extrinsic compression, for instance in compartment syndrome. Arterial dissection can also cause ALI; a history of ripping chest or abdominal pain prior to ischaemia should raise the suspicion of aortic dissection.

\section{$\underline{\text { Clinical Features }}$}

Classically acute limb ischaemia presents with the ' 6 Ps': pain, pallor, pulselessness, perishing cold, paraesthesia and paralysis.

In the early stages the leg is cold and pale due to an absence of venous flow. After 6-12 hours skin mottling occurs, which blanches with pressure as capillaries fill with deoxygenated blood. If left untreated skin mottling becomes fixed as capillaries rupture, distal arteries thrombose and the ischaemia becomes irreversible. Developing neuromuscular ischaemia presents as evolving paraesthesia, paralysis and calf tenderness. The presence of these is an important guide to the urgency of treatment.

Presenting symptoms can vary depending upon the cause of the ischaemia and presence of PAD. In patients with PAD, signs and symptoms of ischaemia are less severe due to collaterals. Typically, patients may describe deterioration in their claudication distance or new onset of ischaemia rest pain ('acute-on-chronic ischaemia').

\section{Assessment}

Clinical assessment has three key aims: establish the diagnosis acute ischaemia, identify a cause and classify the severity of the ischaemia.

History should aim to elicit the nature of the patient's symptoms, screen for history of preexisting PAD and identify other potential causes (e.g. palpations, acute chest pain). The limb should be inspected for pallor, temperature and mottling. Pulses should be palpated and sensiomotor function of the foot and calf muscles tested. Both the anterior and posterior compartments of the calf should be gently compressed. Firm or tender compartments are indicative of muscle ischaemia, which can lead to compartment syndrome. 
Findings should be compared with the contralateral limb. Rapid onset of profound ischaemia with normal pulses in the contralateral limb is indicative of acute embolic limb ischaemia. Likewise, an insidious onset of symptoms and absence of contralateral pulses suggests thrombosis. A palpable mass in the popliteal fossa may indicate a thrombosed popliteal aneurysm. In reality differentiating between thrombotic and embolic causes can be challenging.

The severity of the ischaemia can be classified using the Rutherford Classification (Table 5). 62

The presence of sensorimotor deficit is important guide to the severity of the ischaemia. Partial paraesthesia, paralysis and tender calf muscles indicate an immediately threatened limb that requires emergency revascularisation. Where deficit is complete, usually in the presence of fixed mottling, it is highly likely that ischaemia is irreversible and the limb unsalvageable.

\section{$\underline{\text { Resuscitation }}$}

Initial resuscitation should take place as per the CCRiSP protocol. Arterial blood gas analysis is important to check $\mathrm{pH}$ as muscle ischaemia releases hydrogen ions. An electrocardiogram should be performed to investigate for acute myocardial infarction as a precipitating cause.

Immediate anticoagulation is required to prevent thrombus propagation. Unfractionated heparin has the advantage of having a short half-life, however monitoring of activated partial thromboplastin time (aPTT) is required.

Following diagnosis pre-operative imaging can guide management options, however it should not be undertaken if it will delay revascularisation. This is especially noteworthy in patients requiring transfer to a vascular centre. In this situation imaging should be conducted at the vascular centre so not to delay transfer. 
CTA is investigation of choice due to its rapid availability and non-invasive nature. Although duplex can be effective in ALI it requires an experienced operator and is of limited value in proximal occlusion. ${ }^{63}$ MRA is time consuming and often not available acutely, therefore it is only used if the limb is not threatened.

\section{Definitive management}

As with chronic ischaemia, the definitive management of ALI can be broadly divided into conservative, revascularisation and amputation.

\section{$\underline{\text { Surgical revascularisation }}$}

Surgical revascularisation involves embolectomy with subsequent bypass if results are poor. Initially the femoral bifurcation is identified, vessels controlled and a transverse arteriotomy is created in the common femoral artery. A Fogarty embolectomy balloon catheter is then passed proximally and distally to trawl thrombus. This is repeated until two clear runs in each vessel. 'On-table' angiography is recommended following embolectomy to assess for persistent occlusion and thrombolysis can be instilled into the vessel to aid recanalisation. If femoral embolectomy fails, popliteal embolectomy or bypass can be performed. Occasionally primary bypass is undertaken, particularly in cases of thrombosed popliteal aneurysms.

\section{Endovascular revascularisation}

Percutaneous catheter-directed thrombolysis is an endovascular alternative for revascularisation. For this a wire is inserted into the artery to cross the occlusion and a thrombolysis catheter is parked into the thrombus. This allows for continuous, localised infusion of thrombolytic agents for several hours. Agents such as tissue plasminogen activator or urokinase can be used. Angiography is repeated to check for resolution after a 12-24 hours. A variety of thrombectomy catheters can also be used to aid clearance. Any underlying stenosis exposed during lysis can angioplastied if it is limiting flow.

Both techniques have their relative benefits and drawbacks. Embolectomy can be performed quickly (even under local anaesthesia) and achieve rapid revascularisation. It is of limited value in patients with underlying atherosclerotic disease as the Fogarty 
catheter may not traverse the disease and trawling can lead to further plaque rupture and dissection.

Thrombolysis is of particular use in this situation and allows for management of underlying stenotic plaques during treatment. The technique is percutaneous and considered less invasive then surgery. It does however carry risk of bleeding, stroke and distal embolism, and therefore patient selection is important. Lysis is also slower in clearing thrombus compared with embolectomy and may not be appropriate when emergency revascularisation is required

Results for two meta-analyses shows comparable one year limb salvage and overall mortality rates between both treatments. ${ }^{64,65}$ The decision on technique therefore depends largely on the nature of the ischaemia and fitness for surgery.

\section{Best revascularisation strategy}

The revascularisation strategy for an acutely ischaemic limb depends upon the patient's symptoms, degree of ischaemia and fitness for surgery. The Rutherford classifications can help act as a guide to decision making however patient's rarely fit completely into one category and therefore choosing the best revascularisation strategy can be difficult.

\section{Viable limb (Rutherford 1 - not immediately threatened)}

Clinical findings: Minimal discomfort, intact capillary refill, no paraesthesia/paralysis, audible Doppler signals, no tenderness of anterior/posterior compartments

In cases where the limb is viable conservative management can be trialled and revascularisation may not be required. This situation may be observed in acute-on-chronic ischemia where a patient has deteriorating claudication distance. If symptoms progress to $\mathrm{CLI}$ then revascularisation can be planned in a similar manner to chronic ischaemia.

\section{Threatened limb (Rutherford $2 a-$ prompt treatment required)}

Clinical findings: Pain, delayed capillary refill time, distal paraesthesia (toes/forefoot) but no paralysis, weak/inaudible Doppler signals, no tenderness of anterior/posterior compartments 
This is often due to thrombosis of atherosclerotic disease. In this situation the limb requires revascularisation but time is available to plan appropriate treatment. Surgical embolectomy is unlikely to be successfully due to atherosclerotic disease in this situation. Both thrombolysis or bypass are therefore potential treatment options. Increasingly hybrid procedures using both techniques are utilised. This is often seen in cases of thrombosed popliteal aneurysms where thrombolysis opens tibial run-off vessels for subsequent bypass.

Threatened limb (Rutherford $2 b-$ immediate treatment required)

Clinical findings: Pain, slow capillary refill time, paraesthesia and paralysis of the foot, inaudible Doppler signals, tender and firm anterior/posterior compartments (indicating ischaemia)

This situation is a vascular emergency requiring immediate revascularisation, usually due to embolism. Surgical embolectomy is usually preferred as thrombolysis may not achieve timely revascularisation.

Irreversible ischaemia (Rutherford 3 - unsalvageable limb)

Clinical findings: Fixed mottling, profound paraesthesia and paralysis of the foot, inaudible Doppler signals. Pain may be absent due to the paraesthesia.

A limb with complete paralysis, paraesthesia and fixed mottling is irreversibly ischaemic and unsalvageable. Revascularisation in this situation would lead to the massive return of hydrogen and potassium ions into the circulation which can be fatal. In cases of irreversible ischaemia amputation is the only surgical option, although palliation may be appropriate in the frail and co-morbid.

\section{Compartment syndrome and 4 compartment fasciotomies}

Following revascularisation it is always prudent to consider the possibility of compartment syndrome. This arises as reperfusion of ischaemic muscle causes swelling and increased compartment pressures. If not treated this spirals into permanent neurological damage and muscle necrosis. 
In patients with evidence of muscle ischaemia or profound ischaemia, four compartment fasciotomy is mandatory. Muscle should be inspected for viability and necrotic tissue debrided. In other patients a low threshold to perform fasciotomies is required. The diagnosis of compartment syndrome is clinical and compartment pressures or serum creatinine kinase results are unreliable. In patients who are intubated post-operatively this can particularly be challenging. If there is any doubt, it is best to perform.

\section{$\underline{\text { Conclusion }}$}

Acute and chronic limb ischaemia represent a significant part of the workload for vascular surgeons and carry considerable morbidity and mortality for patient. Peripheral arterial disease is the leading cause of both, with smoking, hypertension and hyperlipidaemia key risk factors. Risk factor modification is mandatory in all patients. Treatment should be tailored to the patient's symptoms and anatomy of their disease with open and endovascular revascularisation techniques frequently being employed.

References Marrett E, DiBonaventura M, Zhang Q. Burden of peripheral arterial disease in Europe and the United States: a patient survey. Health Qual Life Outcomes 2013;11(1):175.

2 Waton S, Johal A, Heikkila K, Cromwell D, Boyle J, Loftus I. National Vascular Registry: 2017 Annual report. London: The Royal College of Surgeons of England, November 2017.

3 Fowkes FGR, Rudan D, Rudan I, Aboyans V, Denenberg JO, McDermott MM, et al. Comparison of global estimates of prevalence and risk factors for peripheral artery disease in 2000 and 2010: A systematic review and analysis. Lancet 2013;382(9901):1329-40..

4 Criqui MH, Aboyans V. Epidemiology of Peripheral Artery Disease. Circ Res 2015;116(9):1509-26.

5 Hart W, Guest J. Critical limb ischaemia: the burden of illness in the UK. Br Med Econ, Vol. 8. 1995. pp. 211-2.

$6 \quad$ Khan S, Flather M, Mister R, Delahunty N, Fowkes G, Bradbury A, et al. Characteristics and Treatments of Patients with Peripheral Arterial Disease Referred to UK Vascular Clinics: Results of a Prospective Registry. Eur J Vasc Endovasc Surg 2007;33(4):442-50.

7 Emdin CA, Anderson SG, Callender T, Conrad N, Salimi-Khorshidi G, Mohseni H, et al. Usual blood pressure, peripheral arterial disease, and vascular risk: cohort 
study of 4.2 million adults. BMJ 2015;351:h4865.

Fowkes FG, Housley E, Cawood EH, Macintyre CC, Ruckley C V, Prescott RJ.

Edinburgh Artery Study: prevalence of asymptomatic and symptomatic peripheral arterial disease in the general population. Int J Epidemiol 1991;20(2):384-92..

9 Selvin E, Erlinger TP. Prevalence of and risk factors for peripheral arterial disease in the United States: Results from the National Health and Nutrition Examination Survey, 1999-2000. Circulation 2004;110(6):738-43.

10 Khaleghi M, Isseh IN, Bailey KR, Kullo IJ. Family history as a risk factor for peripheral arterial disease. Am J Cardiol 2015;114(6):928-32..

11 Pereira C, Miname MH, Makdisse MRP, Watanabe C, Pesaro AE, Jannes CE, et al. Peripheral arterial disease in heterozygous familial hypercholesterolemia. Atherosclerosis 2015;242(1):174-8.

12 Price JF, Mowbray PI, Lee AJ, Rumley A, Lowe GDO, Fowkes FGR. Relationship between smoking and cardiovascular risk factors in the development of peripheral arterial disease and coronary artery disease. Edinburgh Artery Study. Eur Heart $J$ 1999;20(5):344-53..

13 Office for National Statistics. Adult smoking habits in the UK: 2017. London: Office for National Statistics, July 2018.

14 Office for National Statistics. E-cigarette use in Great Britain. London: Office for National Statistics, July 2018.

15 Shahab L, Goniewicz ML, Blount BC, Brown J, McNeill A, Alwis KU, et al. Nicotine, Carcinogen, and Toxin Exposure in Long-Term E-Cigarette and Nicotine Replacement Therapy Users: A Cross-sectional Study. Ann Intern Med 2017;166(6):390-400.

16 Diabetes UK. State of the Nation: Challenges for 2015 and beyond. London: Diabetic UK, 2014.

17 Hirsch AT. Peripheral Arterial Disease Detection, Awareness, and Treatment in Primary Care. Jama 2001;286(11):1317..

18 Dickinson PJ, Carrington AL, Frost GS, Boulton AJM. Neurovascular disease, antioxidants and glycation in diabetes. Diabetes Metab Res Rev 2002;18(4):26072.

19 Jude EB, Oyibo SO, Chalmers N, Boulton AJM. Peripheral arterial disease in diabetic and nondiabetic patients: A comparison of severity and outcome. Diabetes Care 2001;24(8):1433-7.

20 Spreen MI, Gremmels H, Sprengers RW, Verhaar MC, Statius van Eps RG, de Vries JP. Diabetes is association with decreased limb survival in patients with critical limb ischaemia: pooled data from two randomised controlled trial. Diabetes Care. 
2016;39(11):2058-2064.

21 Humphries MD, Brunson A, Hedayati N, Romano P, Melnkow J. Amputation risk in paients with diabetes mellitus and peripheral artery disease using statewide data. Ann Vasc Surg. 2016;30:123-31

22 Planas A, Clará A, Pou JM, Vidal-Barraquer F, Gasol A, de Moner A, et al. Relationship of obesity distribution and peripheral arterial occlusive disease in elderly men. Int J Obes Relat Metab Disord 2001;25(7):1068-70.

23 Gorter PM, Olijhoek JK, Van Der Graaf Y, Algra A, Rabelink TJ, Visseren FL. Prevalence of the metabolic syndrome in patients with coronary heart disease, cerebrovascular disease, peripheral arterial disease or abdominal aortic aneurysm. Atherosclerosis 2004;173(2):363-9.

24 Rantner B, Kollerits B, Pohlhammer J, Stadler M, Lamina C, Peric S, et al. The fate of patients with intermittent claudication in the 21st century revisited-results from the CAVASIC Study. Sci Rep 2017;8:45833.

25 Norgren L, Hiatt WR, Dormandy JA, Nehler MR, Harris KA, Fowkes FGR, et al. InterSociety Consensus for the management of peripheral arterial disease (TASC II). Int Angiol 2007;26(2):82-157.

26 Hardman RL, Jazaeri O, Yi J, Smith M, Gupta R. Overview of classification systems in peripheral artery disease. Semin Intervent Radiol 2014;31(4):378-88.

27 National Institute for Health and Care Excellence. Peripheral arterial disease: diagnosis and management. London: National Institue for Health and Care Excellence, 2018.

28 The Royal College of Radiologists. Standards for Intravascular Contrast Administration to Adult Patients Third Edition. London: The Royal College of Radiologists, 2015.

29 Collaboration AT. Collaborative meta-analysis of randomised trials of antiplatelet therapy for prevention of death, myocardial infarction, and stroke in high risk patients. BMJ 2002;324(7329):71-86.

30 Wong PF, Chong LY, Mikhailidis DP, Robless P, Stansby G. Antiplatelet agents for intermittent claudication. Cochrane Database Syst Rev 2011;(11):CD001272.

31 Gent M. A randomised, blinded, trial of clopidogrel versus aspirin in patients at risk of ischaemic events (CAPRIE). Lancet 1996;348(9038):1329-39.

32 Hiatt WR, Fowkes FGR, Heizer G, Berger JS, Baumgartner I, Held P, et al. Ticagrelor versus clopidogrel in symptomatic peripheral artery disease. N Engl J Med 2017;376(1):32-40.

33 Anand S, Bosch J, Eikelboom J, Connolly S Diaz R, Widlimsky P et al. Rivaroxaban with or without aspirin in patients with stable peripheral or carotid artery disease: 
an international, randomised, double-blind, placebo-controlled trial. Lancet, 2018; 391(10117):219-229

34 Kumbhani DJ, Steg PG, Cannon CP, Eagle KA, Smith SC, Goto S, et al. Statin therapy and long-term adverse limb outcomes in patients with peripheral artery disease: insights from the REACH registry. Eur Heart J 2014;35(41):2864-72.

35 Mohler ER, Hiatt WR, Creager MA. Cholesterol reduction with atorvastatin improves walking distance in patients with peripheral arterial disease. Circulation 2003;108(12):1481-6.

36 Bonaca MP, Nault P, Giugliano RP, Keech AC, Pineda AL, Kanevsky E, et al. Lowdensity lipoprotein cholesterol lowering with evolocumab and outcomes in patients with peripheral artery disease: insights from the FOURIER trial (further cardiovascular outcomes research with PCSK9 inhibition in subjects with elevated risk). Circulation. 2018;137(4):338-350.

37 National Institute for Health and Care Excellence. Hypertension in adults: diagnosis and management: Guidance and guidelines. London: National Institute of Health and Care Excellence, 2016.

38 National Institute for Health Care and Excellence (NICE). Smoking: harm reduction. London: National Institute of Health and Care Excellence, 2013.

39 Rosenberg L, Palmer JR, Shapiro S. Decline in the risk of myocardial infarction among women who stop smoking. N Engl J Med 1990;322(4):213-7..

40 Lane R, Harwood A, Watson L, Leng GC. Exercise for intermittent claudication. Cochrane Database Syst Rev, 2017;12:CD000990..

41 Vemulapalli S, Dolor RJ, Hasselblad V, Schmit K, Banks A, Heidenfelder B, et al. Supervised vs unsupervised exercise for intermittent claudication: A systematic review and meta-analysis. Am Heart J 2015;169(6):924-37.

42 Hageman D, Fokkenrood HJP, Gommans LNM, van den Houten MML, Teijink JAW. Supervised exercise therapy versus home-based exercise therapy versus walking advice for intermittent claudication. Cochrane Database Syst Rev 2018;4.

43 De Backer TLM, Vander Stichele R, Lehert P, Van Bortel L. Naftidrofuryl for intermittent claudication. Cochrane Database Syst Rev 2008;2:CD001368.

44 Vietto V, Franco JVA, Saenz V, Cytryn D, Chas J, Ciapponi A. Prostanoids for critical limb ischaemia. Cochrane Database Syst Rev 2018;1:CD006544.

45 Golledge J, Moxon J V., Rowbotham S, Pinchbeck J, Yip L, Velu R, et al. Risk of major amputation in patients with intermittent claudication undergoing early revascularization. Br J Surg 2018;105(6):699-708.

46 Taylor GI, Palmer JH. The vascular territories (angiosomes) of the body: experimental study and clinical applications. Br J Plast Surg 1987;40(2):113-41. 
47 Bradbury AW, Adam DJ, Beard JD, Cleveland T, Forbes JF, Fowkes FGR, et al. Bypass versus angioplasty in severe ischaemia of the leg (BASIL): Multicentre, randomised controlled trial. Lancet 2005;366(9501):1925-34.

48 Khalil AA, Boyd A, Griffiths G. Interposition vein cuff for infragenicular prosthetic bypass graft. Cochrane Database Syst Rev 2012;9:CD007921.

49 Wilasrusmee C, Siribumrungwong B, Horsirimanont S, Poprom N, Jirasiritham J, Thakkinstian A. Clinical results of biologic prosthesis: A systematic review and meta-analysis of comparative studies. Ann Med Surg 2017;15:26-33.

50 Bolia A, Brennan J, Bell PRF. Recanalisation of femoro-popliteal occlusions: Improving success rate by subintimal recanalisation. Clin Radiol 1989;40(3):325

51 Bradbury AW, Adam DJ, Bell J, Forbes JF, Fowkes FGR, Gillespie I. Bypass versus Angioplasty in Severe Ischaemia of the Leg (BASIL) trial: An intention-to-treat analysis of amputation-free and overall survival in patients randomized to a bypass surgery-first or a balloon angioplasty-first revascularization strategy. J Vasc Surg 2010;52(6):1751..

52 Bradbury AW, Adam DJ, Bell J, Forbes JF, Fowkes FGR, Gillespie I, et al. Bypass versus Angioplasty in Severe Ischaemia of the Leg (BASIL) trial: Analysis of amputation free and overall survival by treatment received. J Vasc Surg 2010;51(5 Suppl):

53 Meecham L, Patel S, Bate GR, Bradbury AW. A Comparison of Clinical Outcomes Between Primary Bypass and Secondary Bypass After Failed Plain Balloon Angioplasty in the Bypass versus Angioplasty for Severe Ischaemia of the Limb (BASIL) Trial. Eur J Vasc Endovasc Surg 2018;55(5):666-71. 5.

54 Rosenfield K, Jaff MR, White CJ. Trial of a paclitaxel-coated balloon for femoropopliteal artery disease. J Vasc Surg 2016;63(3):846.

55 Kayssi A, Al-Atassi T, Oreopoulos G, Roche-Nagle G, Tan KT, Rayan, DK. Drugeluting balloon angioplasty versus uncoated balloon angioplasty for peripheral arterial disease of the lower limbs. Cochrane Database Syst Rev. 2016;8:CD011319

56 Spreen MI, Martens JM, Hansen BE, Knippenberg B, Verhey E, Van Dijk LC, et al. Percutaneous transluminal angioplasty and drug-eluting stents for infrapopliteal lesions in critical limb ischemia (PADI) trial. Circ Cardiovasc Interv 2016;9(2).

57 CX Symposium. (2018). Paclitaxel-eluting stent fails BATTLE against bare metal stent - CX Symposium. [Online] Available at: https://www.cxsymposium.com/drug-eluting-stent-battle-bare-metal-stent/ [Accessed 19 Sep. 2018].

58 Hunt BD, Popplewell MA, Davies H, Meecham L, Jarrett H, Bate G, et al. BAlloon versus Stenting in severe Ischaemia of the Leg-3 (BASIL-3): Study protocol for a randomised controlled trial. Trials 2017;18(1). Doi: 10.1186/s13063-017-1968-6. 
59 Aboyans V, Ricco JB, Bartelink MLEL, Björck M, Brodmann M, et al. 2017 ESC Guidelines on the Diagnosis and Treatment of Peripheral Arterial Diseases, in collaboration with the European Society for Vascular Surgery (ESVS). Eur J Vasc Endovasc Surg 2018;55(3):305-68..

60 Howard DPJ, Banerjee A, Fairhead JF, Hands L, Silver LE, Rothwell PM. PopulationBased Study of Incidence, Risk Factors, Outcome, and Prognosis of Ischemic Peripheral Arterial Events. Circulation 2015;132(19):1805-15..

61 Campbell WB, Ridler BMF, Szymanska TH. Current management of acute leg ischaemia: Results of an audit by the Vascular Surgical Society of Great Britain and Ireland. Br J Surg 1998;85(11):1498-503.

62 Suggested standards for reports dealing with lower extremity ischemia. Prepared by the Ad Hoc Committee on Reporting Standards, Society for Vascular Surgery/North American Chapter, International Society for Cardiovascular Surgery. J Vasc Surg 1986;4(1):80-94.

63 Ascher E, Hingorani A, Markevich N, Schutzer R, Kallakuri S. Acute lower limb ischemia: The value of duplex ultrasound arterial mapping (DUAM) as the sole preoperative imaging technique. Ann Vasc Surg 2003;17(3):284-9.

64 Wang JC, Kim AH, Kashyap VS. Open surgical or endovascular revascularization for acute limb ischemia. J Vasc Surg 2016;63(1):270-8.

65 Darwood, R, Berridge DC, Kessel DO, Robertson I, Forster R. Surgery versus thrombolysis for initial management of acute limb ischaemia. Cochrane Database Syst Rev 2018;8:CD002784 
$\underline{\text { Tables }}$

1. Risk factors of PAD

\begin{tabular}{|l|l|}
\hline Non - Modifiable & Modifiable \\
\hline Age & Smoking \\
\hline Ethnicity & Hypertension \\
\hline Family history & Hypercholesterolaemia \\
\hline & Diabetes mellitus \\
\hline & Chronic kidney disease \\
\hline & Obesity \\
\hline
\end{tabular}

2. Ankle brachial pressure index (ABPI)

\begin{tabular}{|c|c|}
\hline ABPI & Interpretation \\
\hline$>1.2$ & Incompressible, calcified vessels \\
\hline $0.9-1.2$ & Normal \\
\hline $0.6-0.9$ & Mild PAD \\
\hline $0.3-0.6$ & Moderate PAD \\
\hline$<0.3$ & Severe PAD \\
\hline
\end{tabular}

3. The Fontaine Classification of PAD

\begin{tabular}{|c|c|c|}
\hline Stage & Definition & \\
\hline 1 & Asymptomatic & \\
\hline 2 & Intermittent claudication & $2 \mathrm{a})>200 \mathrm{~m}$ \\
\hline 3 & Rest pain & $2 \mathrm{~b})<200 \mathrm{~m}$ \\
\hline 4 & Necrosis and gangrene & Critical limb ischaemia \\
\hline
\end{tabular}


4. Cause of acute limb ischaemia

\begin{tabular}{|c|r|c|}
\hline Thrombotic & Embolic & Other \\
\hline Atherosclerosis & Cardiac arrhythmias & Intimal dissection \\
\hline Occlusion of bypass graft & Cardiac mural thrombus & Trauma \\
\hline Popliteal aneurysm & $\begin{array}{r}\text { Aneurysm of proximal } \\
\text { vessel (e.g. popliteal } \\
\text { aneurysm) }\end{array}$ & latrogenic \\
\hline $\begin{array}{c}\text { Hypercoagulable states (e.g. } \\
\text { thrombophilias) }\end{array}$ & Atherosclerotic emboli & External compression \\
\hline & latrogenic & \\
\hline
\end{tabular}

5. Rutherford Classification of acute limb ischaemia (http://mrcemb.wikidot.com/peripheral-ischaemia)

\begin{tabular}{|c|c|c|c|c|}
\hline & Capillary return & Motor & Sensory & $\begin{array}{c}\text { Arterial Doppler } \\
\text { signals }\end{array}$ \\
\hline 1 -Viable & V & v & v & v \\
\hline $\begin{array}{c}2 \mathrm{2a}- \\
\text { Threatened } \\
\text { (prompt } \\
\text { treatment } \\
\text { required) }\end{array}$ & Intact/slow & $\mathrm{v}$ & $\begin{array}{l}\text { Partial (toes/distal } \\
\text { fore-foot) }\end{array}$ & $X$ (often) \\
\hline $\begin{array}{c}\text { 2b- } \\
\text { Threatened } \\
\text { (immediate } \\
\text { treatment } \\
\text { required) }\end{array}$ & Slow/absent & $\begin{array}{l}\text { Partial } \\
\text { paralysis }\end{array}$ & $\begin{array}{l}\text { Partial (proximal to } \\
\text { forefoot) or } \\
\text { complete }\end{array}$ & X (Usually) \\
\hline 3 -Irreversible & Fixed staining & $\begin{array}{l}\qquad \mathrm{X} \\
\text { Profound } \\
\text { paralysis }\end{array}$ & $\begin{array}{c}\mathrm{x} \\
\text { Profound }\end{array}$ & $x$ \\
\hline
\end{tabular}




\section{Figures}

1. Critical limb ischaemia of the right foot demonstrating dry necrosis (hallux $-3^{\text {rd }}$ toes) and rubor. The $4^{\text {th }}$ toes is dusky and is likely to develop necrosis. (http://teachmesurgery.com/vascular/peripheral/chronic-ischaemia/)

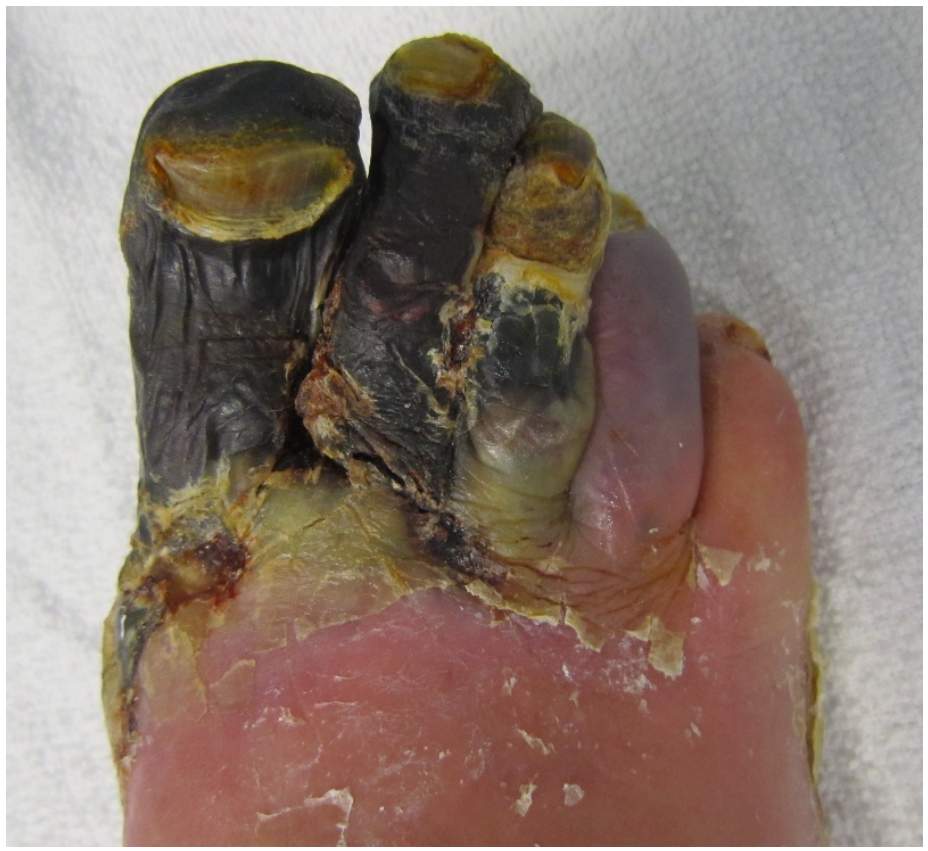

2. CT angiogram (coronal view) -

a) Normal Study

b) Diffuse bilateral disease with multiple right superficial femoral artery (SFA) occlusions and a long occlusion of the left SFA with previous SFA to anterior tibial artery bypass. 

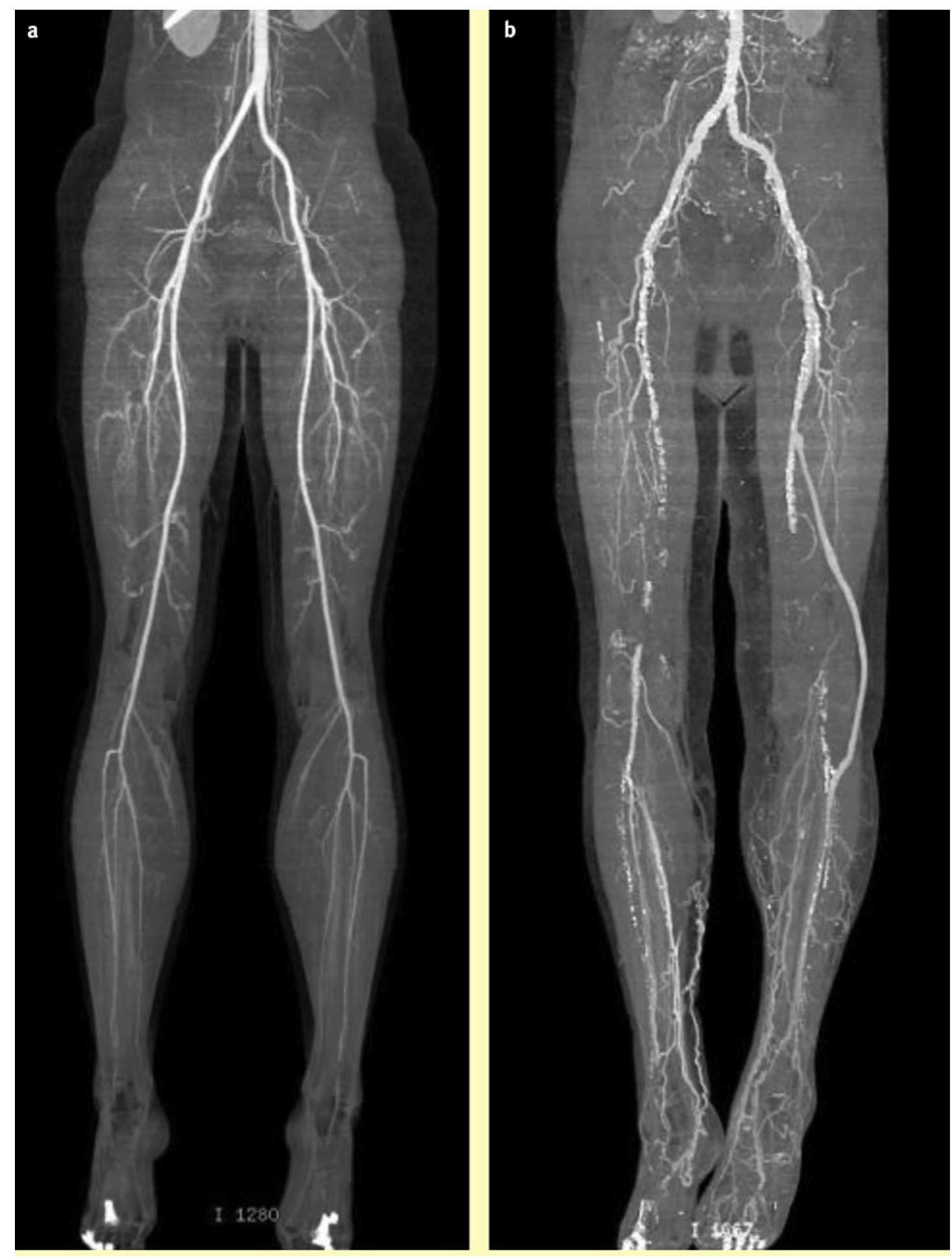

3. Common femoral endarterectomy:

a. Exposure of the artery and arteriotomy. The arteries are slung with silastic slings to provide additional control.

b. Completion of patch repair.

(http://teachmesurgery.com/consent/vascular/femoral-endarterectomy/) 

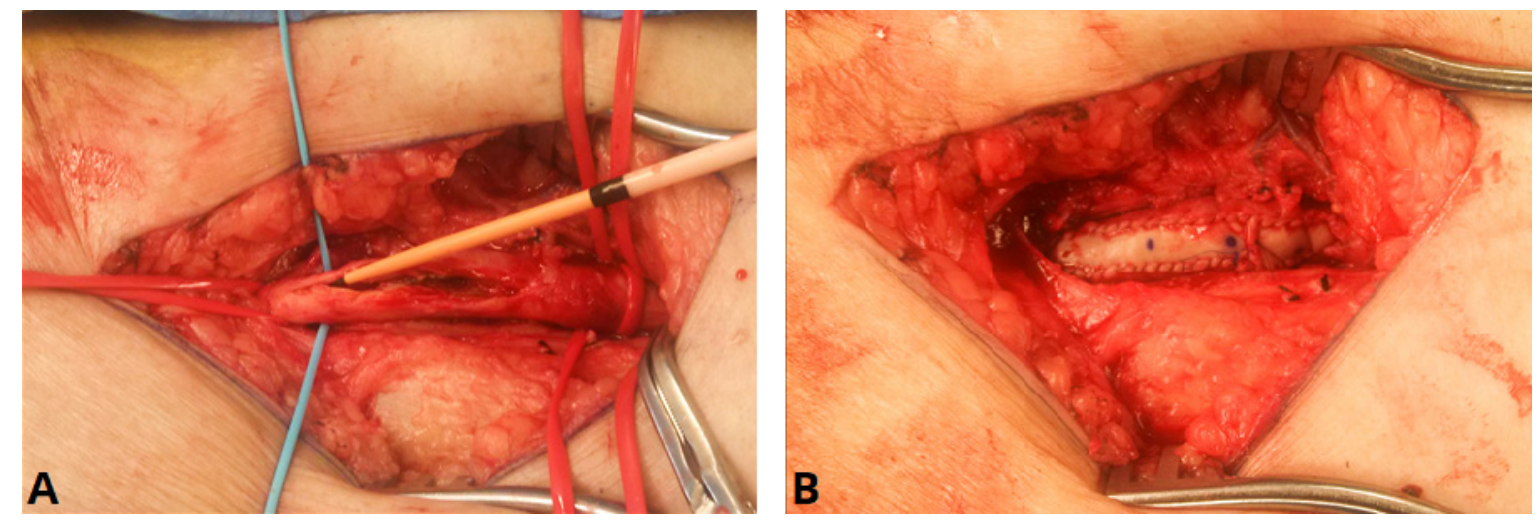

4. Digital subtraction angiogram of the aortic bifurcation and iliac system: Left - Occlusion of the right common iliac artery with collateralisation Right - Completion angiogram following insertion of stent into the right common iliac artery.

(https://www.bmj.com/content/320/7238/854)

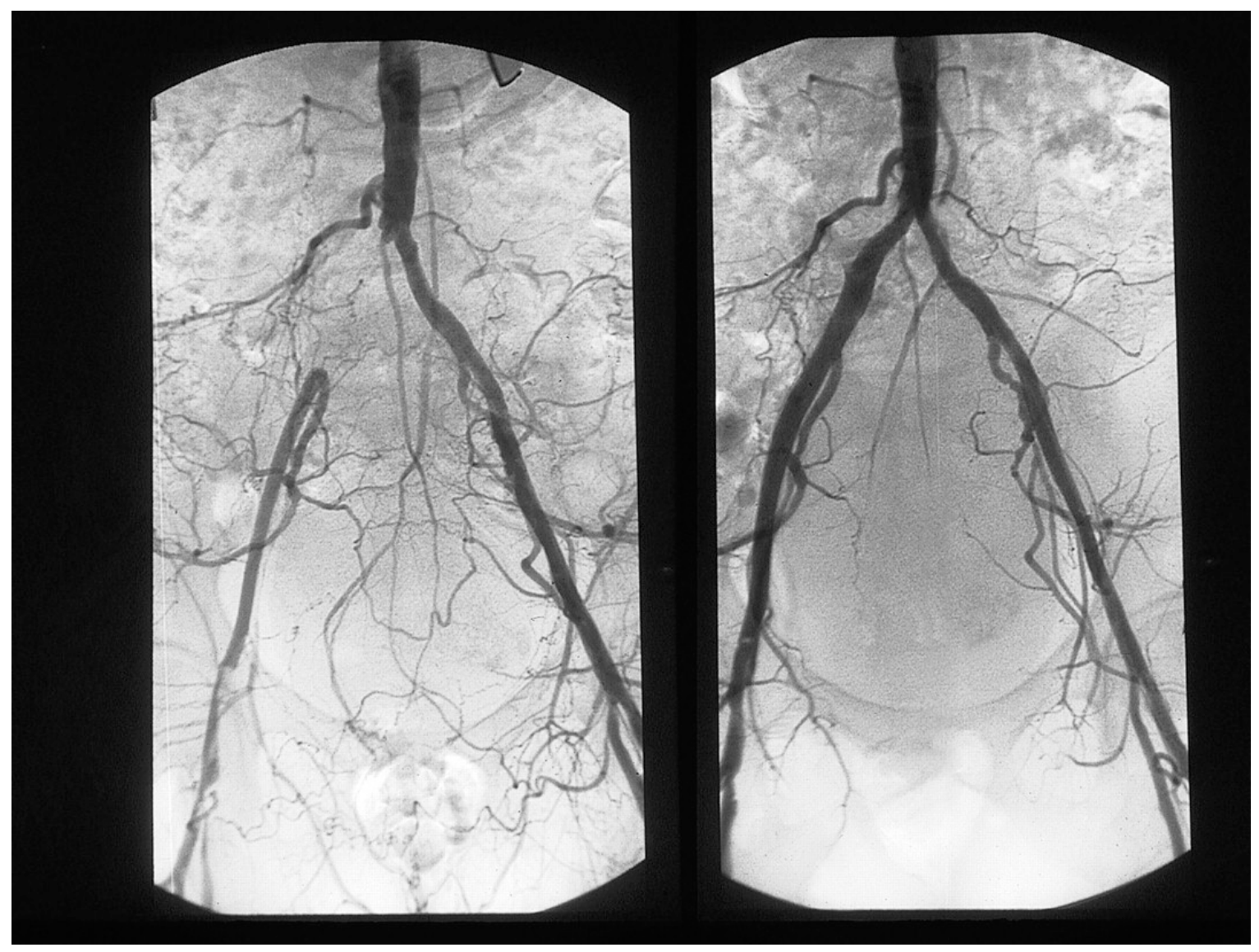

5. Below knee amputation demonstrating the Burgess flap technique 
(http://www.lifebeyond4limbs.com/recovering-from-a-lower-limb-amputation/)
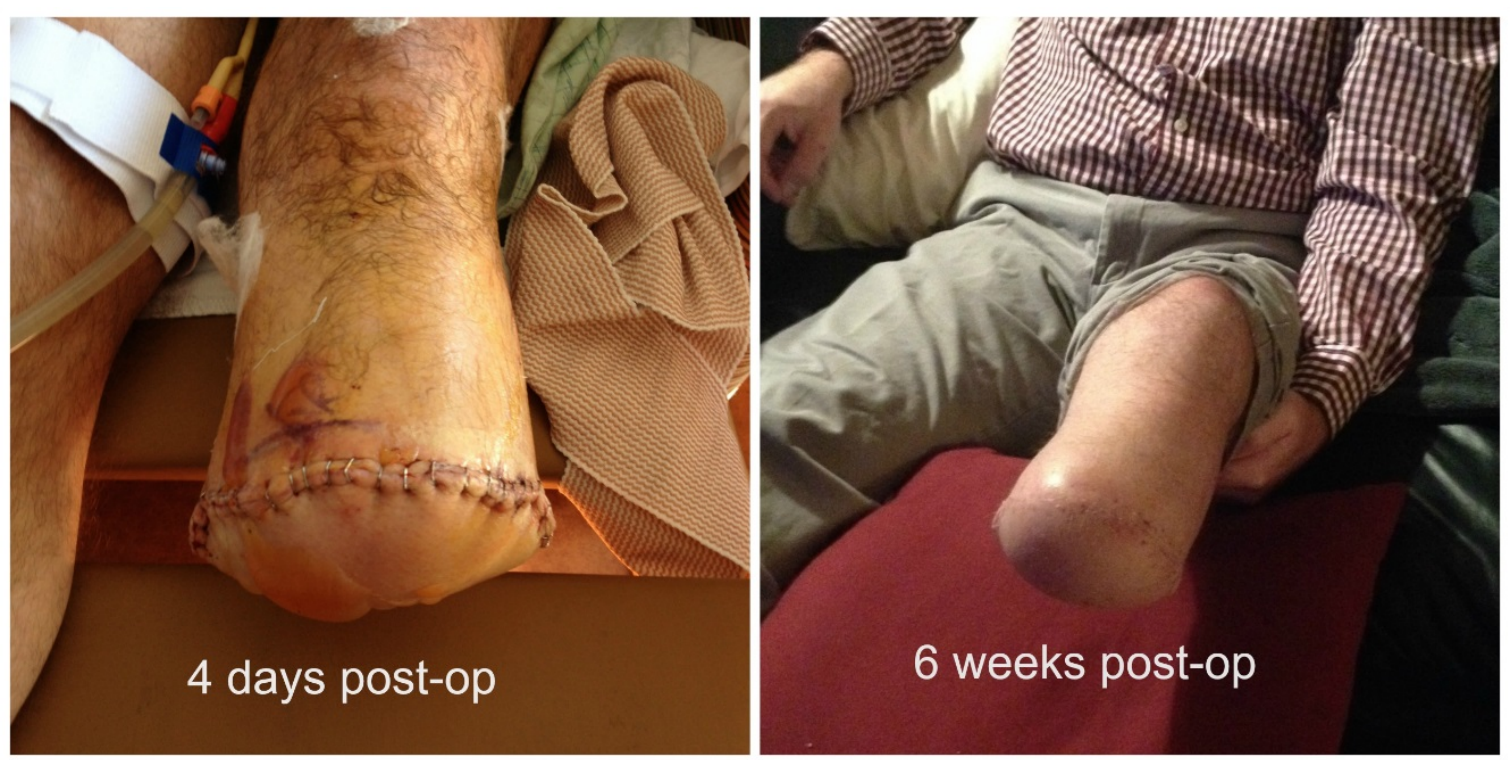

6. Blue toe syndrome of the right hallux (http://heelhurt.com/blog/15-blue-toe-syndrome)

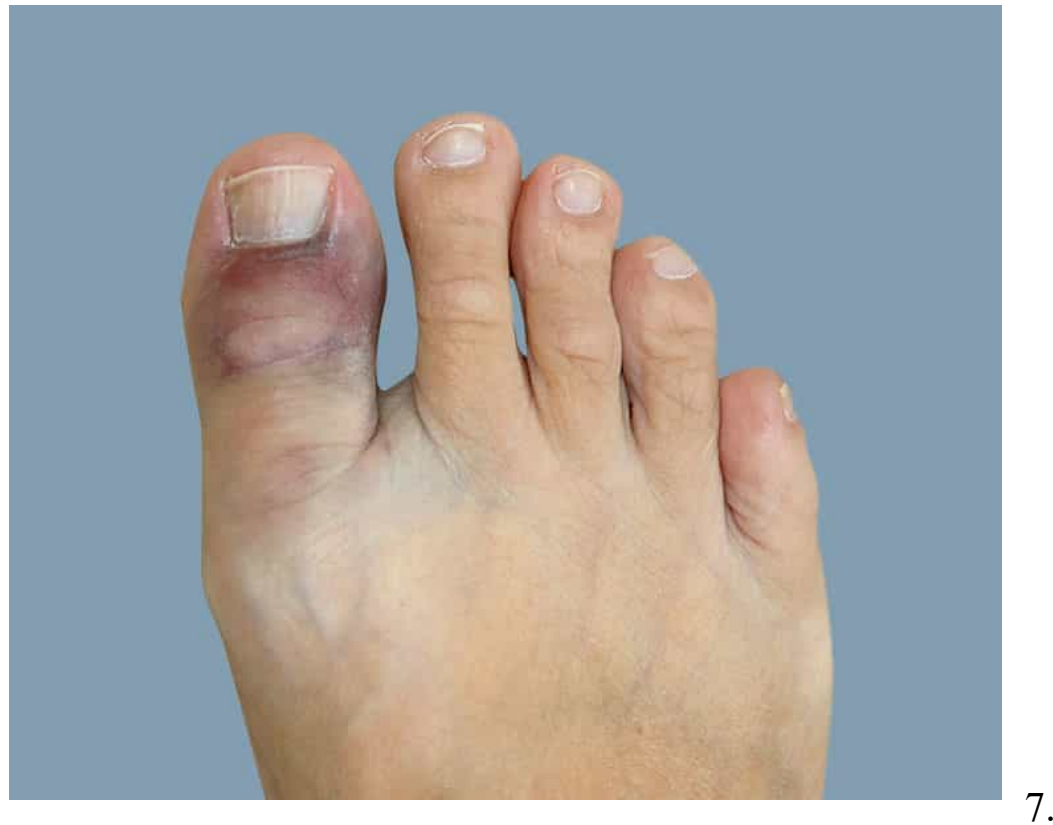

